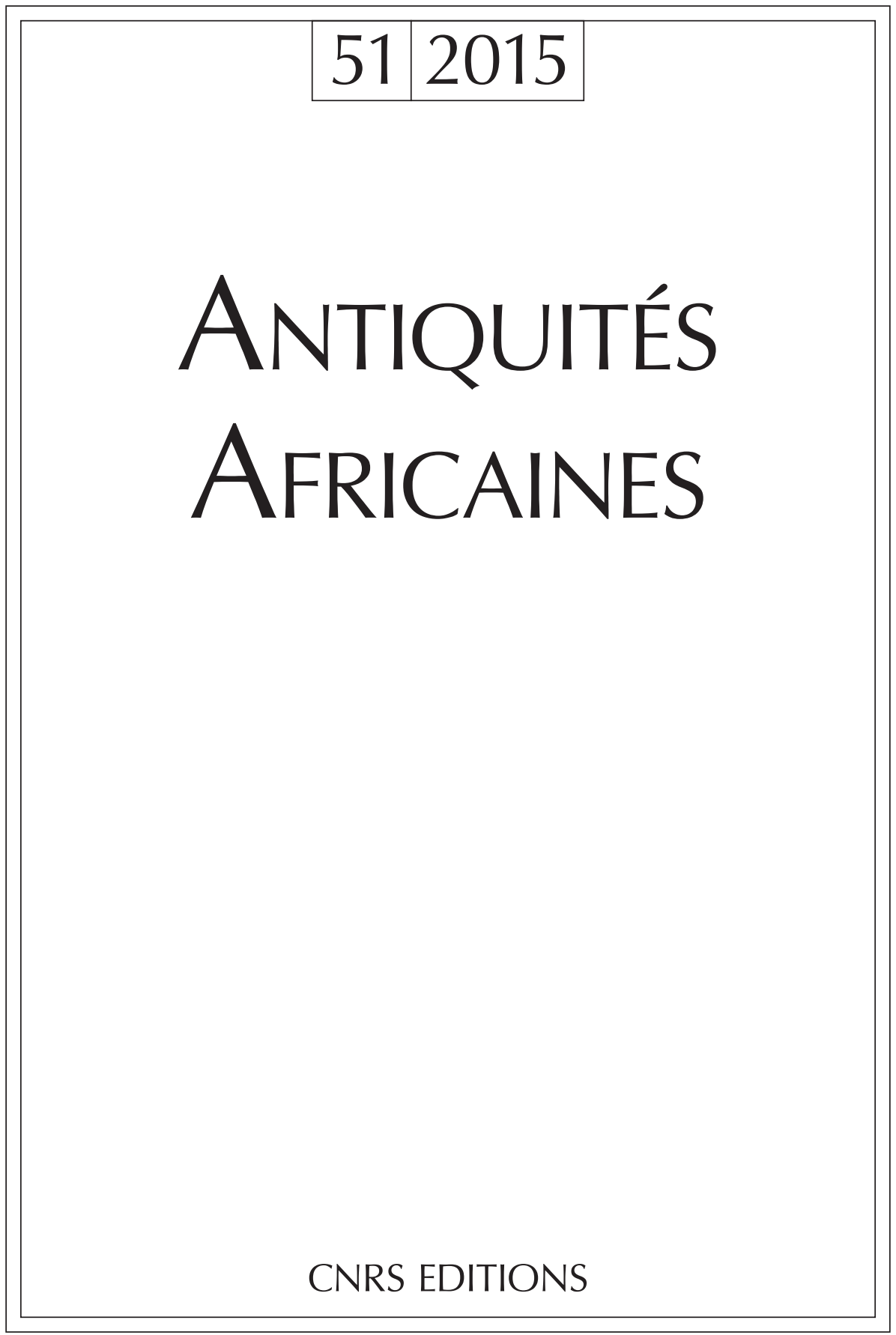




\section{Antiquités africaines, 51 | 2015}

\section{sommaire}

In memoriam Claude Lepelley (1934 - 2015), par Xavier Dupuis ..................................................................... 5

In memoriam Carlos Posac Mon (1922 - 2015) y la arqueología norteafricana, par Enrique Gozalbes Cravioto.

Jihen NACEF, Un atelier de potier à la périphérie de la ville de Thapsus aux premiers siècles de l'Empire, avec une annexe de Claudio CAPELL et Siwar BAKlouti, Caractérisation pétrographique de céramiques provenant d'un possible dépotoir de potier à Thapsus

Ali CHérIF, Données nouvelles sur l'histoire d'une cité méconnue du Haut-Tell tunisien : la ciuitas A[---] / Aïn M'dhoja (région de Makthar)....

Zheira KASDI, Quand l'Amazone fait le Maure : la représentation de la Maurétanie dans le monnayage d'Hadrien

Lotfi NadDarl, Decennalia et Vicennalia d'Antonin le Pieux dans les provinces romaines d'Afrique ?....... 91

Khaled MARMOURI, Une nouveauté pour la carrière de P. Alfius Maximus Numerius Auitus 111

Patrice Faure et Philippe Leveau, Les marges de la Numidie romaine à la lumière d'une nouvelle inscription des Monts des Ouled Naïl.

Michel Bonifay, Mongi Nasr, Yves Rigolr, Jean-Paul Ambrosı et Céline Brun, Le poinçon-matrice de sigillée africaine de Sidi Aïch redécouvert

DOSSIER «À L'ORIGINE DES AMPHORES ROMAINES D'AFRIQUE, II »

Pierre Excoffon et Emmanuel Pellegrino, Amphores africaines du type Ostia XXIII à Fréjus, avec une annexe de Claudio CAPELLI, Analyse pétrographique d'amphores de type Ostia XXIII .

Guillaume DuPERRON et Claudio CAPELL, Observations archéologiques et archéométriques sur quelques types d'amphores africaines en circulation à Arles aux $\|^{\mathrm{e}}$ et Iㅣ $\mathrm{e}$. apr. J.-C.

David Djaoul, Nicolas Garnier et Elisabeth Dodinet, L'huile de ben identifiée dans quatre amphores africaines de type Ostia LIX provenant d'Arles : difficultés d'interprétation

Michel Bonifay, Emmanuel Botte, Claudio Capelu, Alessia Contino, David Djaou, Clementina Panella et André TCHERNIA, Nouvelles hypothèses sur l'origine et le contenu des amphores africaines Ostia LIX et XXIII

NOTES ET CHRONIQUES

Moheddine Chaoval, Cornelia Fortunata, flaminique du culte impérial à Mustis (Tunisie) 213

Martina ANDREOL, Glass finds from a Late Antique-Byzantine farm at Aïn Ouassel (Tunisia) 


\title{
LE POINÇON-MATRICE DE SIGILLÉE AFRICAINE DE SIDI AïCH REDÉCOUVERT
}

\author{
Michel BonifaY", Mongi NasR ${ }^{* *}$, Yves RigolR ${ }^{* * *}$, \\ Jean-Paul AMBROSi ${ }^{* * * *}$ et Céline BRUN ${ }^{* * * * *}$
}

\begin{abstract}
Mots-clés : sigillée africaine ; outil de potier ; archéométrie ; Afrique interne ; période romaine tardive.

Résumé : La redécouverte, dans les collections du Musée Muséum départemental à Gap, du poinçon-matrice de Sidi Aïch permet de corriger et de compléter la description qui en avait été donnée par René Cagnat en 1888 et d'en tenter une première approche archéométrique en XRF portable. Ce poinçon est le témoignage de l'activité d'un grand centre producteur de sigillée africaine récemment réétudié sur le terrain, tout autant que du développement des régions internes de l'Afrique durant l'époque romaine tardive.
\end{abstract}

Keywords: African Red Slip ware; potter's tool; archaeometry; inland Africa; late Roman period.

Abstract: The rediscovery of the Sidi Aïch decorative punch in the Gap departemental museum's collection allows us to correct and complement the description given by René Cagnat in 1888, and to attempt a first archaeometrical approach (handheld XRF). This punch is a testimony to the potters' activity of a major ARS production centre recently restudied in the field, as well as to the economic development of the inland regions of Africa during the late Roman period.
« Un voyageur qui désire garder l'anonyme... ». Ainsi commence la brève communication que René Cagnat consacre, en 1888, à l'atelier de sigillée africaine de Sidi Aïch, dans laquelle il présente quatre objets trouvés sur ce site par un mystérieux correspondant de la Commission d'Afrique du Nord $^{1}$. Trois sont des fragments de plats, non illustrés mais

* Chargé de Recherche au CNRS, Centre Camille Jullian (Aix Marseille Université, CNRS, Ministère de la Culture et de la Communication, INRAP, UMR 7299, Aix-en-Provence).

** Enseignant à l'Université de Sfax, Tunisie.

*** Chercheur indépendant.

**** Chargé de Recherche au CNRS, Centre européen de Recherche et d'Enseignement des Géosciences de l'Environnement, CEREGE (Aix Marseille Université, UMR CNRS 7330, IRD, CDF, Aix-en-Provence). ***** Ingénieur au CNRS, Laboratoire Archéologie et Archéométrie (Maison de l'Orient et de la Méditerranée, UMR 5138, Lyon).

Nous remercions chaleureusement Mme Frédérique Verlinden, directrice, conservatrice en chef du Musée Muséum départemental des Hautes-Alpes à Gap, pour nous avoir autorisés à étudier et publier cet objet. Notre gratitude va également et tout particulièrement à Philippe Borgard (CNRS, CCJ) et à Laurence Pinet (Musée départemental de Préhistoire corse et d'Archéologie à Sartène, précédemment archéologue départementale, Cellule alpine de Recherches archéologiques des Hautes-Alpes) qui ont attiré notre attention sur la collection Aubert-Buès et ont si libéralement partagé avec nous leur connaissance de ce fonds et des conditions de sa création. Merci également à Audrey Copetti (chargée de mission, cellule alpine de Recherches archéologiques) pour la prise en charge du poinçon après étude. Enfin, merci à Claudio Capelli pour sa contribution à l'interprétation des données archéométriques. dont il est dit que deux portent des décors imprimés, tandis que le quatrième est un « cachet en terre cuite » ayant servi à réaliser ces types de décors. Un dessin de ce poinçon-matrice est reproduit « à sa grandeur naturelle » (fig. 1). Dès cette date, Cagnat perçoit bien tout l'intérêt de cette découverte susceptible d'aider à " reconnaître, parmi tous les vases que l'on possède, soit en Afrique, soit ailleurs, les produits provenant de la fabrique de Sidi Aïch ».

Le poinçon-matrice de sigillée africaine de Sidi Aïch a fait date dans l'historiographie de la céramologie africaine. En effet, avant de connaître de récents progrès ${ }^{2}$, l'étude de la technologie et de l'organisation des ateliers de potiers africains est restée longtemps défavorisée par rapport à celle de leurs homologues italiques, gaulois ou hispaniques. De nos jours encore, la plupart des poinçons décoratifs de sigillée africaine sont conservés dans des collections privées sans que leur lieu de découverte ne puisse être précisé3. C'est pourquoi, en dépit du caractère succinct de sa publication initiale, cet objet, l'un des rares découverts in situ en Afrique, a souvent été cité et illustré ${ }^{4}$, alors même que toute trace physique en avait été perdue.

1. Cagnat R., Sidi Aïch, 1888, p. 473.

2. Voir notamment : Mackensen M., El Mahrine, 1993 ; ID., Arbeitsgeräte, 1998 ; ID., Technology and Organisation, 2009.

3. Ex. : Bussière J., Poinçons-matrices, 2008.

4. Atlante I, pl. XVI, 28 ; Mackensen M., El Mahrine, 1993, p. 38, fig. 6. 


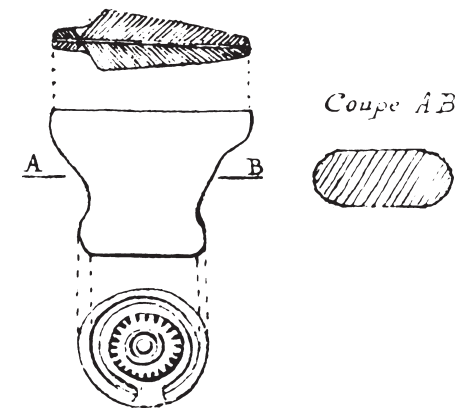

Fig. 1 : Poinçon-matrice de Sidi Aïch (Cagnat R., Sidi Aïch, 1888, sans échelle).

La redécouverte fortuite de ce poinçon dans le fonds du Musée Muséum départemental de Gap permet aujourd'hui d'amender et de compléter la publication qui en avait été faite il y a 128 ans et de replacer cet outil dans le contexte des nouvelles études réalisées sur l'atelier de potiers de Sidi Aïch.

\section{LE CONTEXTE DE CONSERVATION :}

\section{LA COLLECTION AUBERT-BUĖS}

C'est à l'occasion de l'exposition sur la « Collection Aubert-Buès », organisée au Musée Muséum départemental à Gap du 26 janvier au 25 mars 2007, avec le concours d'Yves Chiaramella, que le poinçon de Sidi Aïch est réapparu et a été reconnu comme tel par l'un de nous (M.B.).

La collection Aubert-Buès est l'une des plus importantes collections archéologiques du Maghreb antique conservées en France. Riche de près de 580 pièces, dont plus de 480 vases et lampes en céramique, elle a été réunie jusqu'en 1901 par Clément Aubert (1848-1932) durant les trente années de son activité comme ingénieur puis directeur de la Compagnie des Chemins de Fer de Bône à Guelma. Transmise par héritage à son neveu Jean Buès (1884-1972), elle a été donnée par ce dernier en 1971 à la Société d'Études des Hautes-Alpes qui en a fait dépôt en 1983 au musée de Gap. Lors de la première donation, cette collection avait attiré l'attention de Paul-Albert Février qui soulignait « l'intérêt pour l'histoire régionale de conserver l'unité de cette collection en la faisant entrer dans une collection publique locale $»$. Il relevait également que les objets «paraissaient tous provenir de la région frontalière algérotunisienne : Feriana, Morsott, Haïdra ${ }^{5} »$.

De fait, l'inventaire préliminaire des céramiques de cette collection effectué par Philippe Borgard en 1982 permet de reconnaître une majorité de productions de l'Afrique interne. La plupart des formes de sigillées

5. Lettre de Paul-Albert Février au conservateur des Archives départementales des Hautes-Alpes en date du 7 juin 1971, citée par Laurence Pinet dans le dépliant Collection Aubert-Buès accompagnant l'exposition de 2007 au Musée Muséum départemental des Hautes-Alpes.

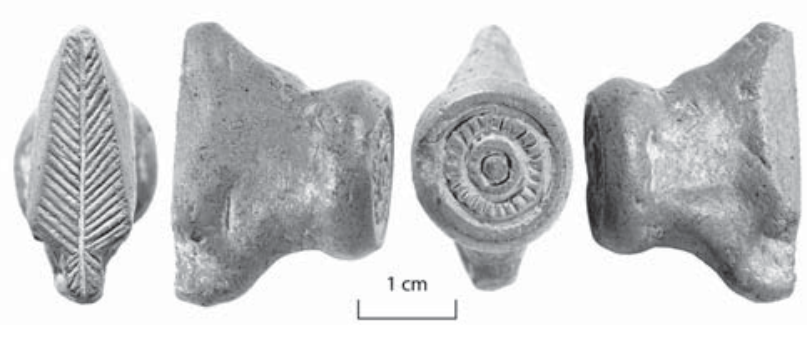

Fig. 2 : Poinçon-matrice de Sidi Aïch. Éch. 1:1 (cliché Y. Rigoir).

africaines présentes dans la collection se rattachent à la typologie des « Other African Wares » de J.W. Hayes ${ }^{6}$, dont l'origine est attribuable aux ateliers continentaux de Tunisie occidentale ou d'Algérie orientale ${ }^{7}$. Il n'y a rien d'étonnant, en conséquence, d'identifier dans cet ensemble le poinçon découvert dans les années 1880 à Sidi Aïch par un voyageur « désirant garder l'anonyme » mais qui pourrait bien avoir été Clément Aubert lui-même.

\section{LE POINÇON : NOUVELLES OBSERVATIONS}

Un seul coup d'œil suffit pour assurer l'identification du poinçon de la collection Aubert-Buès avec celui publié par René Cagnat. Le profil "en enclume » de l'outil, l'association de motifs décoratifs différents, une palmette et une rouelle, sur ses deux faces opposées, la cassure d'un court segment du cercle extérieur de la rouelle ne laissent planer aucun doute à ce sujet. Bien que marquée par la raideur propre au dessin technique de la fin du XIX ${ }^{\mathrm{e}}$ s., la figure publiée en 1888 est relativement précise. Une nouvelle observation du poinçon permet cependant de corriger certains points de cette représentation graphique et de compléter la description de l'objet (fig. 2).

\section{Dimensions}

Il convient tout d'abord d'en rectifier les dimensions. Bien que le texte de Cagnat indique que l'objet est reproduit «à sa grandeur naturelle», on observe que l'éditeur du $B C T H$ a fait subir au dessin une réduction d'environ $75 \%$. En réalité, les mesures du poinçon de Sidi Aïch sont les suivantes :

- hauteur : $2 \mathrm{~cm}$

- longueur de la palmette : 2,6 cm

- diamètre max. de la rouelle : $1,7 \mathrm{~cm}$.

6. Hayes J.W., Late Roman Pottery, 1972, p. 300-304, fig. 58, a-d. 7. Atlante I, p. 140-141 ; BonIfAy M., Études, 2004, p. 51-53. 

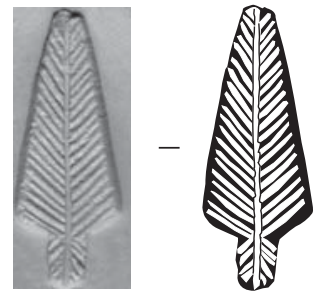
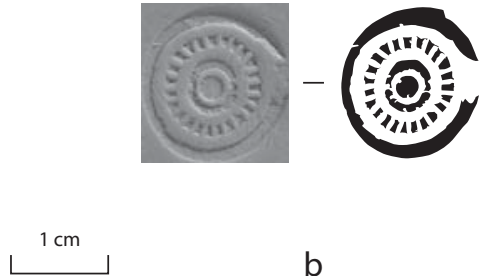

b
Fig. 3 : Impressions du poinçon-matrice de Sidi Aïch : a) palmette ; b) rouelle (M. Bonifay/Y. Rigoir). Éch. 1:1.

\section{MOtIFS DÉCORATIFS}

Difficiles à saisir dans le détail sur le dessin publié en 1888, les motifs décoratifs se présentent ainsi (fig. 3$)^{8}$ :

- Palmette : forme en losange verticalement dissymétrique tronqué et pédonculé. Pointe légèrement ébréchée (?). Dix-neuf nervures latérales divergentes de part et d'autre d'une double nervure axiale. Dans le pédoncule, quatre nervures convergentes à droite, trois à gauche (fig. 3, a).

- Rouelle : forme circulaire. Une pastille centrale entourée d'un cercle, de vingt-trois rayons et d'un autre cercle ébréché sur un huitième de son segment extérieur (fig. 3, b).

\section{DÉtAILS TECHNIQUeS}

Cagnat n'ayant probablement jamais eu en main le poinçon de Sidi Aïch, il n'avait pu en donner une description. - Matière : la pâte de cet objet est de couleur orange, proche de celle utilisée pour la production des sigillées africaines. La surface, apparemment non engobée, est soigneusement lissée mais laisse apparaître, à la binoculaire, de nombreux grains de quartz (éolien).

- Fabrication : il est probable que les deux extrémités du poinçon ont été fabriquées séparément puis jointes entre elles par adjonction d'un tenon modelé. Les traces de collage sont particulièrement nettes du côté de la rouelle, plus discrètes du côté de la palmette.

\section{- Incision des motifs :}

Palmette (fig. 4, a) : il semble que les nervures divergentes et convergentes ont tout d'abord été incisées de manière jointive, en arêtes de poisson ; puis la nervure axiale a été tracée, comme le prouvent les bavures d'argile qui bordent cette dernière ; enfin, la forme losangique de la palmette a été précisée par une découpe du contour à la lame (?).

Rouelle (fig. 4, b) : les deux cercles concentriques paraissent avoir été dessinés à l'aide d'une tige tubulaire, végétale ou métallique (?), dont les fines rayures sont visibles au fond des creux ; il est difficile de savoir si l'incision des rayons est intervenue avant ou après celle des cercles ; le contour extérieur de la rouelle, relativement irrégulier, a été peut-être repris à la lame.

8. Les dessins de cette figure ont été réalisés d'après l'impression des deux faces du poinçon dans de la pâte à modeler. Cette reproduction ne tient pas compte du coefficient de rétractation de l'argile.

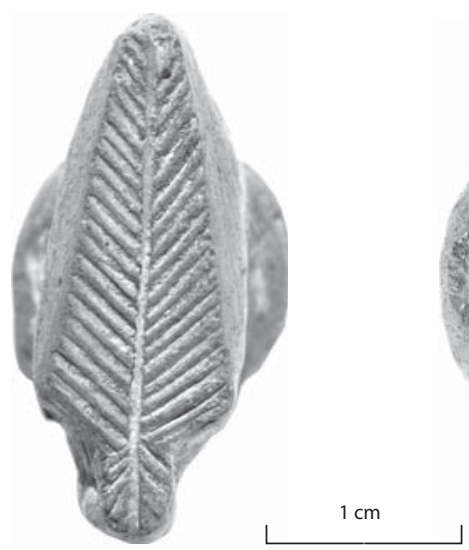

a

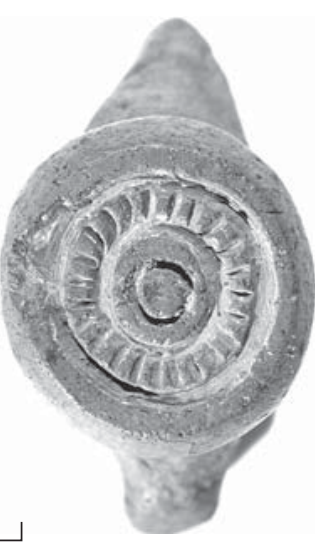

b
Fig. 4 : Détail des deux faces du poinçon. Éch. 2:1 (cliché Y. Rigoir).

\section{Comparaisons}

- Forme du poinçon : l'outil de Sidi Aïch est un rare exemple en Afrique de poinçon-matrice à double face décorative. La plupart des poinçons recensés sur les ateliers de Tunisie ne permettent chacun l'impression que d'un seul motif. Ils sont généralement modelés en forme de cylindre ou bien de cône plus ou moins aplati. Seul un poinçon-matrice découvert à Tiddis partage avec celui de Sidi Aïch cette particularité de pouvoir être utilisé sur deux faces9.

- Motifs : contrairement à ce qui a pu être réalisé avec le poinçon de Tiddis, il n'a pas été possible de recenser au sein du mobilier recueilli en prospection à Sidi Aïch, ni sur aucun site de consommation, de fragment de plat ayant été décoré avec le poinçon publié en 1888. Certains exemples de palmettes ou de rouelles recueillies sur le site se rapprochent des motifs incisés sur le poinçon mais aucun n'est réellement identique $^{10}$. On ne peut pas exclure, toutefois, que la rouelle ait été ébréchée au moment même de la fabrication du poinçon et que le poinçon n'ait finalement jamais été utilisé. - Style décoratif : on remarque que les palmettes pédonculées sont typiques des styles décoratifs B et C de J.W. Hayes qui les attribue aux ateliers de la Tunisie centrale et méridionale. Par ailleurs, la rouelle est très particulière. La disposition des rayons à l'intérieur, entre deux cercles concentriques, et non pas en périphérie de ceux-ci la distingue bien des productions du nord de la Tunisie, par exemple celle des ateliers d'El Mahrine ${ }^{11}$ ou de Sidi Khalifa ${ }^{12}$. Cette variante est en revanche attestée sur une rouelle de plus petite dimension (D. : 1,2 cm) du style C, probablement une sigillée de la catégorie $\mathrm{E}^{13}$. Les comparaisons pointent ainsi d'une part vers la technologie des ateliers de l'Afrique interne (Tiddis), d'autre part vers le style décoratif des ateliers du Sud tunisien.

9. Hayes J.W., Late Roman Pottery, 1972, p. 296.

10. NASR M., Recherches, 1992 et notamment NASR M., Sigillée claire africaine, 2005, p. 221-223 et pl. XLIII-XLIV.

11. Mackensen M., El Mahrine, 1993, pl. 5-7, poinçons 14 à 31.

12. Ben Moussa M., La production de sigillées africaines, 2007, fig. 69-70, poinçons 8 à 28 .

13. Hayes J.W., Late Roman Pottery, 1972, p. 237 et fig. 39cc, type 40 ; ID., Athenian Agora XXXII, 2008, pl. 58, 1195 (P 7508). 


\begin{tabular}{|c|c|c|c|c|c|c|c|c|c|c|c|c|c|c|c|c|c|c|c|}
\hline \multirow{2}{*}{\multicolumn{2}{|c|}{ chantillon }} & \multicolumn{2}{|c|}{$\mathrm{Al}$} & \multicolumn{2}{|c|}{$\mathrm{Si}$} & \multicolumn{2}{|c|}{$\mathrm{P}$} & \multicolumn{2}{|c|}{$S$} & \multicolumn{2}{|c|}{ K } & \multicolumn{2}{|c|}{$\mathrm{Ca}$} & \multicolumn{2}{|c|}{$\mathrm{Ti}$} & \multicolumn{2}{|c|}{$\mathrm{Mn}$} & \multicolumn{2}{|l|}{ 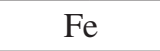 } \\
\hline & & $\mathrm{m}$ & $\sigma$ & $\mathrm{m}$ & $\sigma$ & $\mathrm{m}$ & 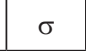 & $\mathrm{m}$ & $\sigma$ & $\mathrm{m}$ & 0 & $\mathrm{~m}$ & 0 & $\mathrm{~m}$ & 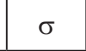 & $\mathrm{m}$ & $\sigma$ & $\mathrm{m}$ & $\sigma$ \\
\hline \multirow{3}{*}{$\begin{array}{c}\text { ACD } \\
397\end{array}$} & & 27,03 & 0,81 & 85 & 0,69 & 0,12 & 0,06 & 0,24 & & 1 & 77 & 12 & 02 & 1,76 & 0,02 & 0,06 & 0,005 &, 12 & \\
\hline & face & 39 & & 4 & 3 & 2 & 6 & 47 & & 5 & 6 & 2 & 3 & 66 & 02 & 06 & & & 03 \\
\hline & & 4 & & & 4 & & 6 & 8 & & 05 & & & & 1,58 & & 07 & & & 03 \\
\hline \multirow{3}{*}{$\begin{array}{l}\text { A } \\
3\end{array}$} & & 2 & & & ) & & 5 & 1 & & & & & & 66 & & 5 & & & \\
\hline & 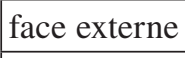 & 19,35 & c & 7 & 0,74 & 0,12 & 0,06 & 0,37 & 0 & 8,50 & 0, & 30 & 3 & 1,39 & 0,02 & 0,04 & & & ,02 \\
\hline & and & 3,02 & 0 & 5 & 0,80 & 0,13 & 0,06 & 0,18 & 0 & 5. & 0 , & 55 & 0,02 & 1,49 & 0,02 & 0,05 & 0 & 38 & 0,02 \\
\hline \multirow{3}{*}{$\begin{array}{c}\text { SA } \\
15\end{array}$} & e & 25,12 & 0,94 & 75 & 0,70 & 0,13 & 0,07 & 0,59 & 0,05 & 0,63 & 0 , & 3 & 5 & 1,43 & 0,02 & 0,07 & 0 & 4 & 0,03 \\
\hline & face & 25,54 & 0,83 &, 88 & 0,65 & 0,13 & 0,06 & 0,49 & 0,04 &, 00 & 0,08 & 18 & 0,05 & 1,44 & 0,02 & 0,08 & 0,005 & 10,26 &, 03 \\
\hline & & 17,40 & 0,76 & ,99 & 0,74 & 0,18 & 0,06 & 0,04 & 0,04 & 9,07 & 0,08 & 67 & 0,05 & 1,25 & 0,02 & 0,09 & 0,005 & 31 & 0,03 \\
\hline \multirow{3}{*}{$\begin{array}{l}\text { SA } \\
12\end{array}$} & e & 24,41 & 0,81 &, 77 & 0,63 & 0,02 & 0,06 & 0,23 & 0,04 & 4,73 & 0,10 & 18 & 04 & 1,54 & 0,02 & 0,10 & c & 3 &, 03 \\
\hline & face $e$ & 21,75 & 0,75 & 43,33 & 0,62 & 0,05 & 0,06 & 0,76 & 0 & 7 & 0, & 2 & 4 & 1,62 & 0,02 & 0,10 & c & 8 & $\pi$ \\
\hline & & 16,68 & 0, & 9 & 0, & 0 & 0 & 0,06 & & & & 4 & & 1,34 & 0,02 & 0,12 & c & 27 & 0,03 \\
\hline \multirow{3}{*}{ Poinçon } & & 18,96 & 0,7 & 51,60 & 0,70 & 0 , & 0,07 & 0,91 & & 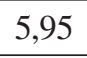 & 0,07 & 11,36 & 0,05 & 1,78 & 0,02 & 0,06 & 0,005 & 11 & 0 \\
\hline & & 19,02 & & 6,16 & 0,65 & 0,33 & 0,08 & 1,16 & & & & 15,76 & & 1,74 & 0,02 & 0,10 & 0,005 & 63 &, 03 \\
\hline & - & 20,04 & 0,72 & 50,85 & 0,64 & 0,26 & 0,07 & 0,72 & 0,05 & 5,41 & 0,06 & 12,45 & 0,05 & 1,74 & 0,02 & 0,08 & 0,005 & 8,40 & 0,0 \\
\hline
\end{tabular}

Tableau 1 : Moyennes $(\mathrm{m})$ et écarts-type $(\sigma)$ des mesures en XRF portable (Bruker Tracer IV SD, valeur moyenne sur trois réplicas en \% des éléments analysés). SA = sigillée africaine, échantillons de référence de l'atelier de Sidi Aïch. ACD = sigillée africaine, échantillons de référence de I'atelier de Sidi Khalifa (J.-P. Ambrosi).

\section{Test archéométrique}

Bien qu'il n'y ait pas de raison particulière de mettre en doute le lieu de découverte de ce poinçon, nous avons jugé intéressant de tester une approche archéométrique sur cet objet. Le problème spécifique du poinçon-matrice de Sidi Aïch est qu'il ne peut subir aucune analyse destructive, même minime. Pour cette raison, nous avons utilisé une méthode non destructive, actuellement en développement, mais dont la validité pour la céramologie n'est pas encore entièrement établie : la spectrométrie de fluorescence X portable (XRF portable) $)^{14}$. De fait, les mesures effectuées sur le poinçon-matrice ${ }^{15}$, sur deux tessons de référence de l'atelier de Sidi Aïch (SA 12 et SA 15) ${ }^{16}$ et sur deux tessons-test d'un atelier du nord de la Tunisie, choisi pour son éloignement géographique ${ }^{17}$ et sa bonne caractérisation géochimique et pétrographique (Sidi Khalifa : ACD 395 et 397$)^{18}$, ne sont que semi-quantitatives du fait, entre autre, des variations texturales possibles des matériaux analysés.

14. Les analyses ont été réalisées par J.-P.A. avec un matériel de type Brucker Tracer IV SD.

15. Trois mesures effectuées : a) "côté » = sur la paroi latérale ; b) « palme » = face côté palmette ; c) « rouelle » = face coté rouelle.

16. SA 12 : forme Stern XXXV; SA 15 : forme Stern VII (= Hayes 68 variante). Collection de référence du Centre Camille Jullian.

17. Des échantillons d'ateliers plus proches, comme ceux de Thelepte (NASR M., Recherches, 1992), n'étaient pas disponibles.

18. ACD 395 : forme Hayes 87A ; ACD 397 : forme Hayes 103 variante. Collection de référence du Centre Camille Jullian. Échantillons précédemment analysés par C. Brun en fluorescence X conventionnelle : Brun C., Étude technique, 2007, fig. 575, et par Cl. Capelli en lame-mince : Bonifay M., Capelli Cl. et Brun C., Approche intégrée, 2012, p. 51.
En valeurs normalisées en \% des éléments analysés, nos mesures sont en bonne correspondance (tableau 1), seuls le phosphore, le soufre et le calcium montrent des teneurs plus fortes pour le poinçon, en relation probable avec un état de surface différent (c'est-à-dire une altération secondaire), que les tessons analysés. Une comparaison entre les valeurs du silicium (Si) et de l'aluminium (Al) (fig. 5) fait apparaître une assez nette distinction des deux ateliers et, d'autre part, ne contredit pas l'appartenance du poinçon à l'atelier de Sidi Aïch. En effet les analyses de Sidi Khalifa se positionnent à des valeurs de $\mathrm{Si}$ et $\mathrm{Al}$ supérieures à celles de Sidi Aïch et du poinçon. Il est également intéressant de remarquer que les analyses sur les faces internes sont à des valeurs de $\mathrm{Si}$ et $\mathrm{Al}$ inférieures à celles des faces externes, les analyses effectuées sur la tranche des objets étant les plus élevées en ces éléments. La présence d'un engobe épuré sur la surface interne, la surface externe relativement polie, et le plus fort pourcentage d'inclusions de quartz et silicates d'aluminium dans la pâte pourraient bien expliquer ces résultats. Cette remarque confirme l'effet de surface et de texture des matériaux en lien avec la profondeur analysée. Bien que cette approche analytique semble prometteuse, il est encore prématuré de vouloir comparer, sur une population aussi faible, ces résultats à ceux obtenus par les méthodes conventionnelles sur des références d'autres ateliers de sigillées africaines ${ }^{19}$. Ce test archéométrique indique simplement une possible compatibilité entre les compositions chimiques du poinçon-matrice et celles des productions de l'atelier de Sidi Aïch.

19. Voir notamment : Mackensen M. et Schneider G., Production Centres, 2002 et 2006 ; Brun C., Étude technique, 2007. Pour Sidi Aïch, voir Bonifay M., Capelli Cl. et Brun C., Approche intégrée, 2012, p. 54-55. 


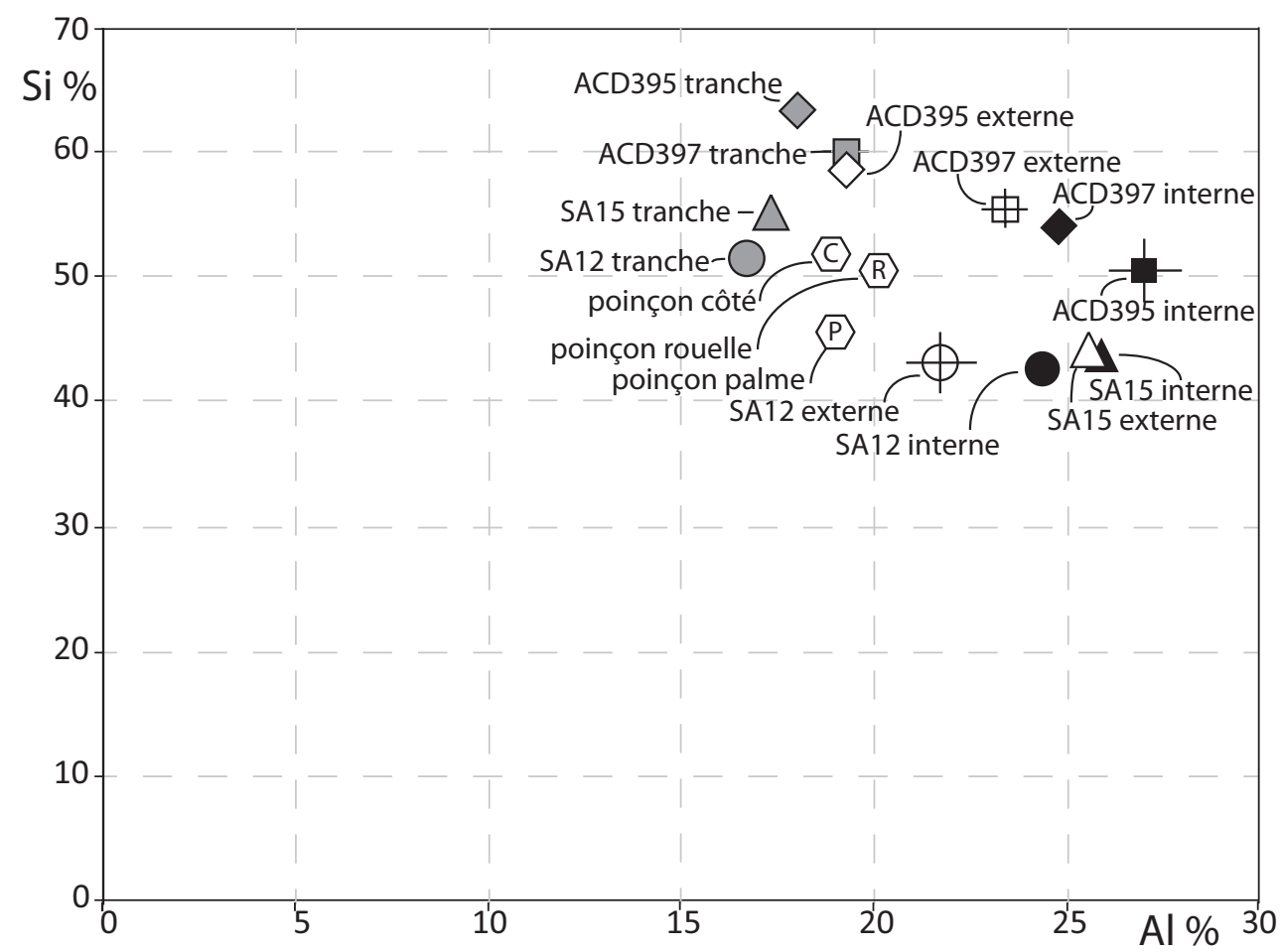

Fig. 5 : Diagramme binaire aluminium - silicium des analyses du poinçonmatrice (trois mesures), de deux échantillons de sigillée africaine de I'atelier de Sidi Aïch (SA 12 et 15) et de deux échantillons de sigillée africaine de I'atelier de Sidi Khalifa (ACD 395 et 397). (Analyses exprimées en \%, cf. tableau 1, la taille des symboles ou les barres verticales et horizontales correspondent aux écarts-type, calculs et graphique J.-P. Ambrosi).

\section{LE CONTEXTE DE DÉCOUVERTE : l'ATELIER DE POTIeRS De Sidı AïCH}

La découverte de ce poinçon-matrice à la fin du XIX ${ }^{\mathrm{e}} \mathrm{s}$. est restée unique sur le site de Sidi Aïch. Aucun autre poinçon n'a pu y être découvert pendant les prospections réalisées en 1966 par Marianne Stern et Jan Willem Salomonson et celles plus récentes conduites par l'un d'entre nous (M.N.).

Rappelons que le site de Sidi Aïch ${ }^{20}$, supposé être le uicus Gemellas ou Gemellae ${ }^{21}$, est situé à environ $36 \mathrm{~km}$ à vol d'oiseau au nord-ouest de la ville de Gafsa, l'antique Capsa (fig. 6). Au-delà de son signalement par Cagnat à la fin du $X^{X}{ }^{e}$ s., l'essentiel de nos connaissances sur l'atelier de potier situé en périphérie de cette agglomération a longtemps reposé sur l'examen comparatif des ateliers de Henchir es-Srira et Sidi Aïch publié par M. Stern en $1968^{22}$. Cette brève étude a fourni un cadre typologique commun aux deux ateliers, qui a servi de base à la plupart des synthèses sur la sigillée africaine continentale qui ont suivi. La fourchette chronologique proposée pour la production de Sidi Aïch a sensiblement évolué : 300 à 450 selon M. Stern, 350-500 selon S. Tortorella et finalement 250(?)-500 selon M. Mackensen ${ }^{23}$. Pour sa part, J.W. Hayes a insisté sur la parenté entre les productions de Sidi Aïch et celles des ateliers de Tunisie centrale et méridionale ${ }^{24}$.

20. Le site couvre plus de 26 ha.

21. Desanges J. et al., Carte des routes et des cités, 2010, p. 149.

22. Stern M., Note analytique, 1968, p. 146 sq.

23. Stern M., Note analytique, 1968, pl. IVb ; Atlante I, 1981, p. 138 ;

Mackensen M., El Mahrine, 1993, p. 38.

24. Hayes J.W., Late Roman Pottery, 1972, p. 300.
La reprise en 1990 des prospections de l'atelier de Sidi Aïch ${ }^{25}$ a donné l'opportunité d'étudier plusieurs zones de fours et d'épandage. La production sur place de vaisselles sigillées est confirmée par la découverte de fragments de casettes de cuisson et celle de lampes par des moules en céramique ${ }^{26}$. L'élaboration de nouvelles typologies (vaisselle de table, poinçons décoratifs, céramique culinaire, lampes $)^{27}$ propres à l'atelier permet de proposer l'hypothèse d'un début de production à la fin $\mathrm{du} \mathrm{II}^{\mathrm{e}} \mathrm{s}$. ou au début du $\mathrm{III}^{\mathrm{e}} \mathrm{s}$. avec des variantes de plats Hayes 27/31 attestés par ailleurs en production A2 et $\mathrm{A} / \mathrm{D}$. Les formes les plus tardives semblent se référer aux grands plats Hayes $90 \mathrm{~A}$ des décennies centrales du VI $\mathrm{siècle}^{28}$. Toutefois la période principale de production, dont le poinçonmatrice se fait le témoin, est comprise entre la fin du $\mathrm{IV}^{\mathrm{e}} \mathrm{s}$. et la fin du $\mathrm{V}^{\mathrm{e}}$ s., comme l'avait déjà souligné J.W. Hayes ${ }^{29}$. C'est l'époque des décors imprimés à motifs géométriques (essentiellement palmes et rouelles) dont la disposition au fond des plats varie selon au moins quatre styles différents. Il est probable que le poinçon-matrice à double face permettait aux potiers de Sidi Aïch de réaliser rapidement les décors dits de « style $1 »^{30}$ : quatre palmettes disposées en croix, pointes vers l'extérieur, cantonnées de quatre rouelles intercalaires (fig. 7). Ce style décoratif rappelle le style Hayes A(ii) de la sigillée africaine D du nord de la Tunisie et pourrait dater, en conséquence, de la fin du $\mathrm{IV}^{\mathrm{e}} \mathrm{s}$. ou de la première moitié du $\mathrm{V}^{\mathrm{e}}$ siècle.

25. NASR M., Recherches, 1992 ; ID., Sigillée claire africaine, 2005.

26. NASR M., Sigillée claire africaine, 2005, pl. XCIX, 1 et pl. C, 2.

27. NAsR M., Sigillée claire africaine, 2005, p. 91-273 et pl. XII-CXIV. 28. Une poursuite de la production jusqu'à la fin du $\mathrm{VI}^{\mathrm{e}} \mathrm{s}$., voire même le début du VII' s., n'est toutefois pas complètement exclue : NASR M., Sigillée claire africaine, 2005, p. 510-511.

29. Hayes J.W., Late Roman Pottery, 1972, p. 300.

30. NASR M., Sigillée claire africaine, 2005, p. 221-223 et pl. XLVI-XLIX. 


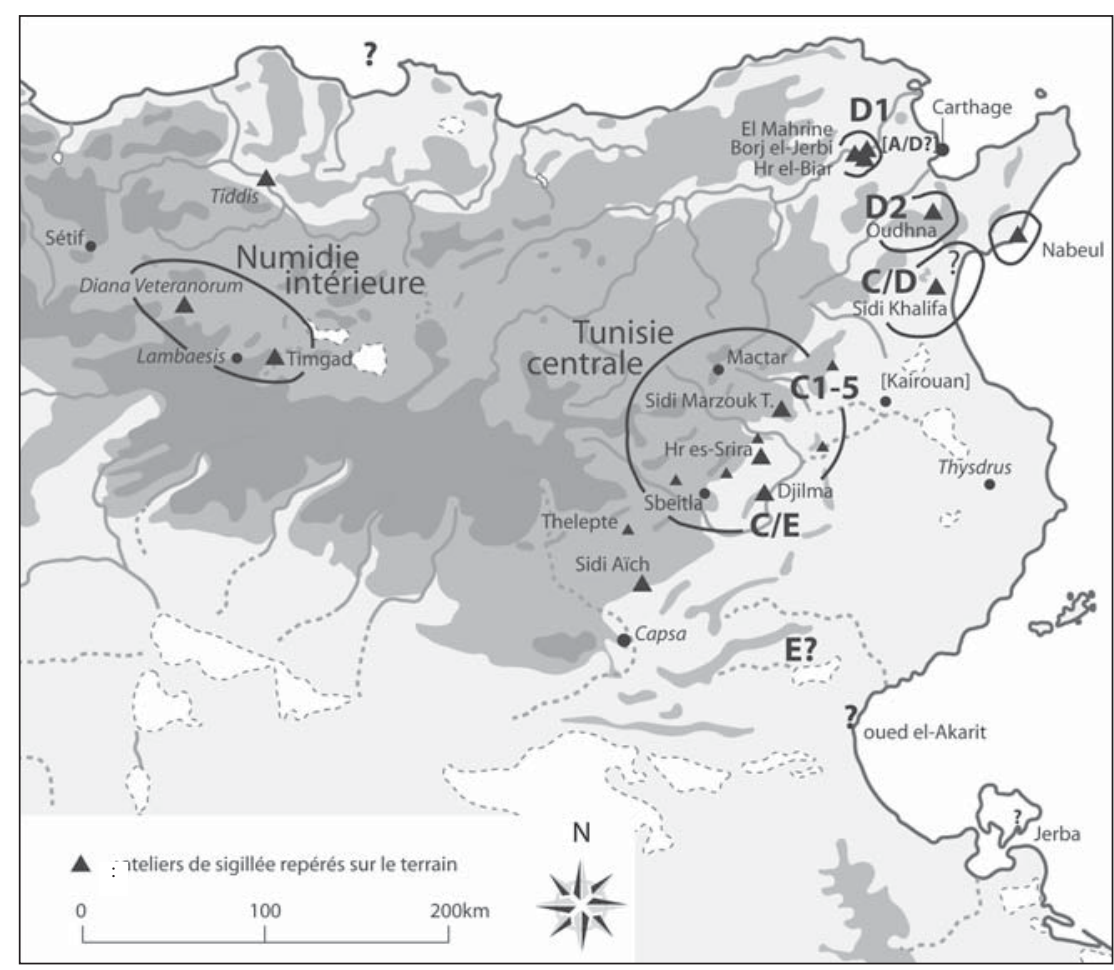

Fig. 6 : Carte de situation de l'atelier de Sidi Aïch (M. Bonifay).

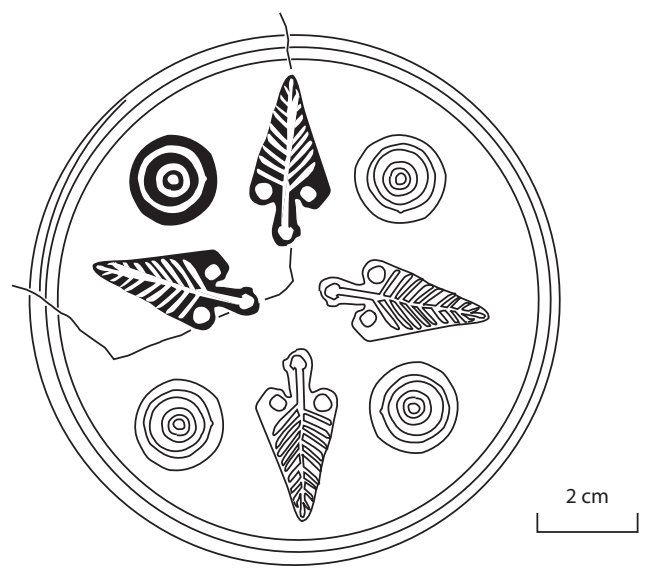

Fig. 7 : Atelier de Sidi Aïch : style décoratif de type 1. Éch. 1:2 (M. Nasr).

\section{Conclusion}

Cette suite donnée, à plus d'un siècle de distance, à l'article de Cagnat sur le poinçon-matrice de Sidi Aïch est une suggestive illustration de la continuité de l'archéologie africaine, du $\mathrm{XIX}^{\mathrm{e}}$ au $\mathrm{XXI}^{\mathrm{e}}$ siècle. Le correspondant anonyme de la Commission d'Afrique du Nord en 1888 est désormais démasqué, probablement en la personne de Clément Aubert ou de l'un de ses collaborateurs. Le lent cheminement de la collection de cet ingénieur des Chemins de Fer, constituée dans les années qui ont précédé ou immédiatement suivi l'instauration du Protectorat français en Tunisie, puis léguée à des héritiers conscients de son importance et enfin dévolue à une collection publique sur intervention d'un grand spécialiste du Maghreb romain, ouvre désormais sur la possibilité de nouvelles études dont la première est consacrée au poinçon-matrice de Sidi Aïch. Ce réexamen, réalisé par une équipe binationale constituée de chercheurs français et tunisien, n'a pas d'autre but que de corriger et compléter l'étude de René Cagnat, en expérimentant des méthodes archéométriques novatrices et en liaison avec les résultats obtenus sur le terrain par l'archéologie tunisienne actuelle.

Par cette note, nous souhaiterions également insister sur l'intérêt de développer les recherches dans les zones internes de l'Afrique romaine. Comme l'a bien démontré l'un d'entre nous ${ }^{31}$, l'atelier de Sidi Aïch n'est pas un atelier

31. NASR M., Sigillée claire africaine, 2005, p. 485-491. mineur destiné à l'approvisionnement de marchés locaux. La production de cet atelier est largement diffusée dans tout l'espace continental des Hautes Steppes tunisiennes et des Hautes Plaines algériennes, jusqu'à Sétif. Elle est l'illustration d'un phénomène général de substitut d'importation qui apparaît dans ces régions à la fin du $\mathrm{II}^{\mathrm{e}} \mathrm{s}$. ou au début du $\mathrm{III}^{\mathrm{e}} \mathrm{s}$. et connaît sa phase d'expansion principale au $\mathrm{IV}^{\mathrm{e}}$ et au $\mathrm{V}^{\mathrm{e}}$ siècles ${ }^{32}$. Le remplacement, dans l'intérieur de l'Afrique romaine, de productions céramiques importées par des productions céramiques régionales qui connaissent à leur tour une diffusion continentale déconnectée des grands courants méditerranéens signale peut-être un changement économique plus global, dans ces espaces, à l'époque romaine tardive. En ce sens, le poinçonmatrice de Sidi Aïch pourrait être considéré comme le témoin non seulement de l'activité d'un important centre potier africain mais également du développement économique de toute une région.

Avril 2015 


\section{BIBLIOGRAPHIE}

Ben Moussa M., 2007, La production de sigillées africaines. Recherches d'histoire et d'archéologie en Tunisie septentrionale et centrale, Barcelone (Instrumenta, 23).

BONIFAY M., 2004, Études sur la céramique romaine tardive d'Afrique, Oxford (BAR Int. Ser., 1301).

BoNifAY M., à paraître, Can we speak of Pottery and Amphora 'Import Substitution' in Inland Regions of Roman Africa?, dans D.J. Mattingly, V. Leitch et F. Cole (éds.), Trans-Saharans: Human Mobility and Identity, Trade, State Formation and Mobile Technologies across the Sahara (1000 BC-AD 1500), II. Trade, Cambridge.

Bonifay M., Capelli Cl. et Brun C., 2012, Pour une approche intégrée archéologique, pétrographique et géochimique des sigillées africaines, dans M. Cavalieri, en collab. avec E. De Waele et L. Meulumans (éds.), Industria apium. L'archéologie : une démarche singulière, des pratiques multiples. Hommages à Raymond Brulet, Louvain, p. 41-62.

BRUN C., 2007, Étude technique des productions de l'atelier de Sidi Khalifa (Pheradi Maius, Tunisie) : céramiques culinaires, sigillées et cazettes, dans M. Bonifay et J.-Chr. Tréglia (éds.), $L R C W 2$, Late Roman Coarse Wares, Cooking Wares and Amphorae in the Mediterranean: Archaeology and Archaeometry, Oxford (BAR Int. Ser., 1662), p. 569-579.

Bussière J., 2008, Nouveaux outils de potiers africains d'époque romaine (IVe-VI ${ }^{\mathrm{e}}$ s.), Facta, 2, p. 89-105.

Cagnat R., 1888, L'atelier de poterie de Sidi-Aïch, BCTH, p. 473-474.

Carandini A. (dir.), Anselmino L., Pavolini C., Saguì L., Tortorella S., Tortorici E., 1981, Atlante delle forme ceramiche, I. Ceramica fine romana nel bacino mediterraneo (medio e tardo impero), Rome (Enciclopedia dell'arte antica, suppl.) (abrégé Atlante I).

Desanges J., Duval N., Lepelley Cl. et Saint-Amans S. (éds.), 2010, Carte des routes et des cités de l'est de l'Africa à la fin de l'Antiquité d'après le tracé de Pierre Salama, Turnhout (Bibliothèque de l'Antiquité tardive, 17).
Hayes J.W., 1972, Late Roman Pottery, Londres.

Hayes J.W., 2008, The Athenian Agora, XXXII. Roman Pottery. FineWares Imports, Princeton.

MaCKensen M., 1993, Die spätantiken Sigillata- undLampentöpfereien von El Mahrine (Nordtunesien), Munich (Münchner Beiträge zur Vor- und Frühgeschichte, 50).

MACKensen M., 1998, Arbeitsgeräte aus dem spätantiken Töpfereizentrum von El Mahrine (Nordtunesien), MDAI(R), 105, p. 431-439.

Mackensen M., 2009, Technology and Organisation of ARS Ware Production-Centres in Tunisia, dans J.H. Humphrey (éd.), Studies on Roman Pottery of the Provinces of Africa Proconsularis and Byzacena (Tunisia). Hommage à Michel Bonifay, Porthmouth (JRA Suppl., 76), p. 17-44.

Mackensen M. et Schneider G., 2002, Production Centres of African red slip Ware (3rd-7th c.) in Northern and Central Tunisia: Archaeological Provenance et Reference Groups based on Chemical Analysis, JRA, 15, p. 121-158.

Mackensen M. et Schneider G., 2006, Production Centres of African red slip Ware (2nd-3rd c.) in Northern and Central Tunisia: Archeological Provenance and Reference Groups based on Chemical Analysis, JRA, 19, p. 163-190.

NASR M., 1992, Recherches sur la céramique rouge-orangé dans la région de Gafsa à l'époque romaine : l'atelier de Sidi Ä̈ch, Certificat d'Aptitude à la Recherche de l'Université de Tunis, non publié.

NASR M., 2005, La sigillée claire africaine de la Byzacène du Sud-Ouest : productions et circuits commerciaux, Thèse de Doctorat de l'Université de Provence, non publiée.

NASR M., à paraître, La céramique du Vicus Gemellae entre Syrtis minor et Sitifis, ou les raisons d'une distribution continentale, dans $D e$ la Syrtis minor au golfe de Gabès, une histoire, un patrimoine, $I V^{e}$ Colloque international (Sfax, 20-22 novembre 2014).

STERn E.M., 1968, Note analytique sur des tessons de sigillée claire ramassés à Henchir es Srira et Sidi Aïch, BABesch, 43, p. 146-154. 